\title{
Structures of Graphene/Cobalt Interfaces in Cobalt-Encapsulated Carbon Nanocapsules
}

\author{
Daisuke Matsuura and Tokushi Kizuka \\ Institute of Materials Science, Graduate School of Pure and Applied Sciences, University of Tsukuba, Ibaraki, \\ Tsukuba 305-8753, Japan \\ Correspondence should be addressed to Tokushi Kizuka, kizuka@ims.tsukuba.ac.jp
}

Received 30 October 2011; Revised 13 January 2012; Accepted 23 January 2012

Academic Editor: Takuya Tsuzuki

Copyright ( 92012 D. Matsuura and T. Kizuka. This is an open access article distributed under the Creative Commons Attribution License, which permits unrestricted use, distribution, and reproduction in any medium, provided the original work is properly cited.

Carbon nanocapsules (CNCs) encapsulating cobalt (Co) nanoparticles with a face-centered-cubic structure were synthesized by a gas-evaporation method and were observed by high-resolution transmission electron microscopy. The Co nanoparticles revealed polyhedral shapes; they were truncated by low-index facets. The surfaces of Co nanoparticles were surrounded by graphene layers. The atomic configuration and interlayer spacing at the graphene/cobalt interfaces were investigated.

\section{Introduction}

Since fullerene $\mathrm{C}_{60}$ was discovered, various studies regarding fullerenes and related materials have been conducted, for example, synthesis, analysis of structures and properties, encapsulation, derivatization, and synthesis of larger fullerene molecules and nanotubes [1-5]. Carbon nanocapsules $(\mathrm{CNCs})$ are giant multiwalled fullerenes having voids in their center [6-10]. As is known in the encapsulation of rare-earth elements in smaller fullerenes, CNCs also encapsulate metal- and carbide nanoparticles, bringing in the inhibition of oxidation and grain coarsening of encapsulating nanoparticles $[6-9,11-19]$. In particular, for the encapsulation of magnetic nanoparticles, we can explore new magnetic properties of nanoparticles [14, 15].

In such CNCs encapsulating nanoparticles, the interfaces between the nanoparticle and graphene layers dictate their structural and chemical properties. For metal/graphene systems, the interfaces have also been focused in application of graphene to electronic devices, in which graphene is contacted with metal electrodes and wires [20-29]. Such metal/ graphene interfaces are two-dimensional internal structures. Thus, transmission electron microscopy (TEM) is a most useful method to investigate them. A common obstacle to the analysis is how to prepare thin specimens for TEM observation. Because interface structures are damaged when the specimens are thinned by mechanical and chemical methods. On the other hand, we can observe metal/graphene interfaces in encapsulating CNCs without thinning processes. In this study, we synthesized cobalt (Co)-encapsulated CNCs by a gas-evaporation method and investigated their interface structures by high-resolution TEM.

\section{Method}

An edge of a carbon rod of $5 \mathrm{~mm}$ in diameter was drilled, and a Co rod was packed in the hole. An edge of another carbon rod was sharpened. The two edges of the two rods were contacted in argon of $2 \times 10^{4} \mathrm{~Pa}$, and an alternating current was applied to heat the contact region. As the current was increased, the temperature of the contact region reached melting temperature, resulting in evaporation [30]. Thus, the contact region was heated by Joule heating by current passing. A smoke comprised of particles was produced by evaporation and moved upward. The smoke was collected on microgrids cooled at liquid nitrogen temperature.

The synthesized particles were observed using the transmission electron microscope (JEOL JEM2100F) with a field 


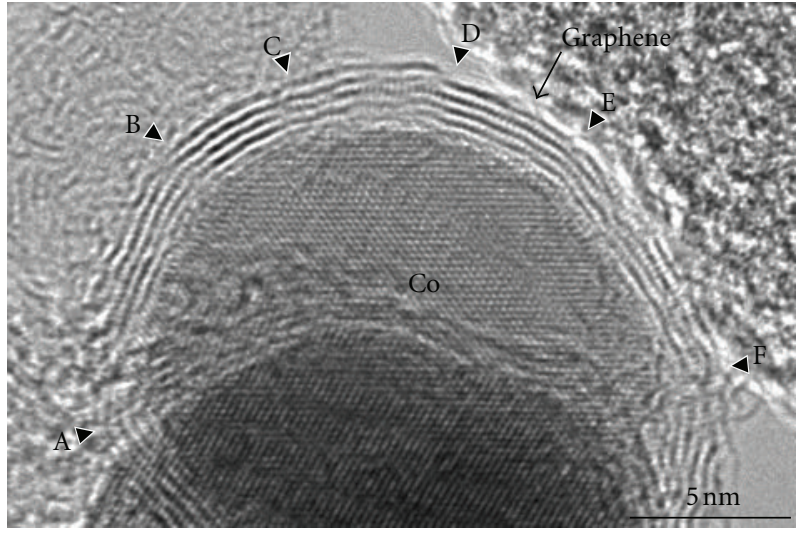

(a)

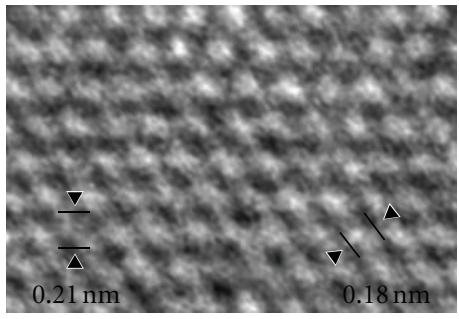

(b)

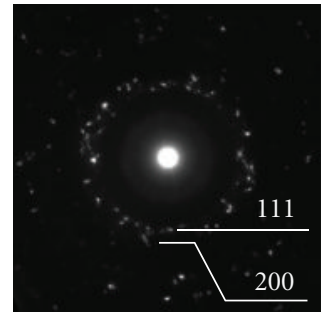

(c)

FIGURE 1: (a) High-resolution image of Co-encapsulated carbon nanocapsule. (b) Enlarged image of the encapsulated Co nanoparticle in (a). (c) Selected-area electron diffraction pattern from aggregate of Co-encapsulated carbon nanocapsules.

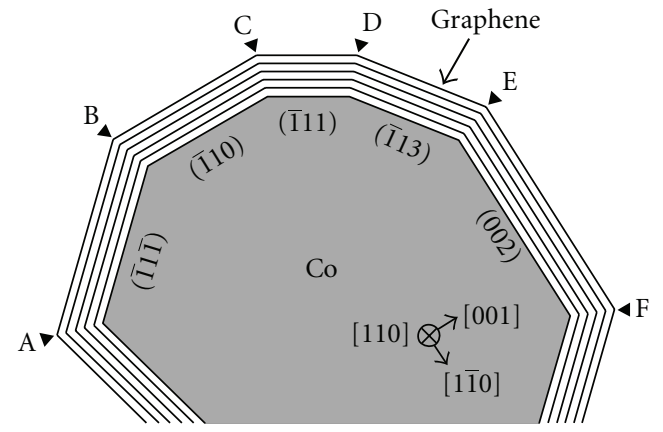

FIGURE 2: Facets of the Co-encapsulated carbon nanocapsule shown in Figure 1(a).

emission electron gun operated at an acceleration voltage of $200 \mathrm{kV}$. The specimens were observed by the lattice imaging under the Scherzer focus conditions.

\section{Results}

Figure 1(a) shows a high-resolution image of a synthesized nanoparticle. In the inner region of the nanoparticle, lattice fringes of $0.21 \mathrm{~nm}$ and $0.18 \mathrm{~nm}$ in spacing are observed, as shown in Figure 1(b). The selected-area electron diffraction from an aggregate of the nanoparticles corresponds to a face-centered-cubic (fcc) structure, as shown in Figure 1(c). From these results, the inner region was identified to be a fcc-Co nanoparticle. The outer region of the nanoparticle is surrounded by graphene layers. Thus, the nanoparticle is a Co-encapsulated CNC. In this study, we examined more than 300 CNCs. The selected-area electron diffraction patterns from the nanoparticles encapsulated in the CNCs were identified as those of fcc-Co nanoparticles. Jiao et al. synthesized Co-encapsulated CNCs by arc discharge and found that the encapsulating Co particles had fcc structures
[31]. Saito also reported that to by similar synthesis of Coencapsulated CNCs, fcc-Co nanoparticles were dominantly produced in addition of small amount of Co nanoparticles with hexagonal-closed-packed structures and $\mathrm{Co}_{3} \mathrm{C}$ nanoparticles [15]. Our result is similar to these previous studies.

The lattice fringes of the Co nanoparticle, that is, $0.21 \mathrm{~nm}$ and $0.18 \mathrm{~nm}$, correspond to $(\overline{1} 11)_{\mathrm{Co}}$ and $(002)_{\mathrm{Co}}$, respectively. Thus, we found the observed direction and the facets of the Co nanoparticle, as shown in Figure 2. Five facets, $(\overline{1} 1 \overline{1})_{\mathrm{Co}},(\overline{1} 10)_{\mathrm{Co}},(\overline{1} 11)_{\mathrm{Co}},(\overline{1} 13)_{\mathrm{Co}}$, and $(002)_{\mathrm{Co}}$, are observed in the upper region of the CNC.

Figure 3 shows a $(0001)_{\text {graphene }} /(\overline{1} 13)_{\text {Co }}$ interface in D-E region in the Co-encapsulated CNC shown in Figure 1(a). The distances between neighboring black dots observed on the graphene layers are $0.21 \mathrm{~nm}$ (Figure 3(a)). In graphene layers, there is no lattice spacing corresponding to this distance. As shown in Figure 4, when graphene layers are viewed

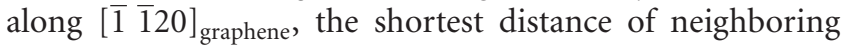
atomic rows is $0.07 \mathrm{~nm}$. Because this distance is smaller than the spatial resolution of the microscope used in this observation, the two neighboring atomic rows separated by $0.07 \mathrm{~nm}$ are observed as one black dot. The distance between the combined black dots is $0.21 \mathrm{~nm}$ (Figure $3(\mathrm{~b})$ ). Thus, the observed black dots of $0.21 \mathrm{~nm}$ in distance on the graphene layers in Figure 3 correspond to the neighboring carbon atomic rows aligned along $[\overline{1} \overline{1} 20]_{\text {graphene }}$ on (0001) graphene

Figure 5 shows the orientational relationship between the graphene layers and Co nanoparticles at the interfaces in region D-E. The enlarged high-resolution image of the interface shown in Figure 3 is shown in Figure 5(a). From this image, we constructed the configuration model of carbon and Co atoms along the direction parallel to the interfaces. To confirm the matching of the lattice image and the model, the overlapping of them is presented in Figure 5(b). The extracted atomic configuration is shown in Figure 5(c). The atomic configuration model at the interface 


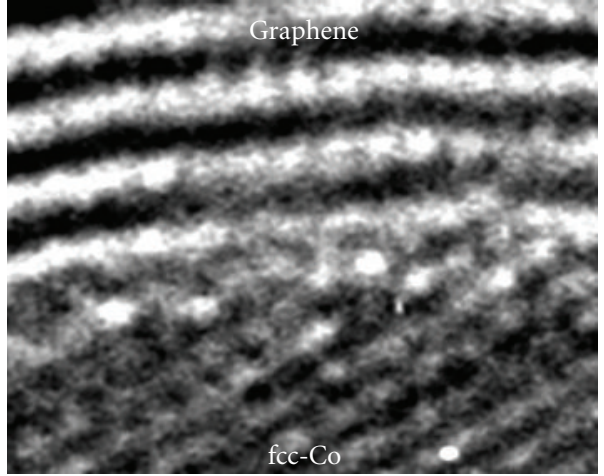

(a)

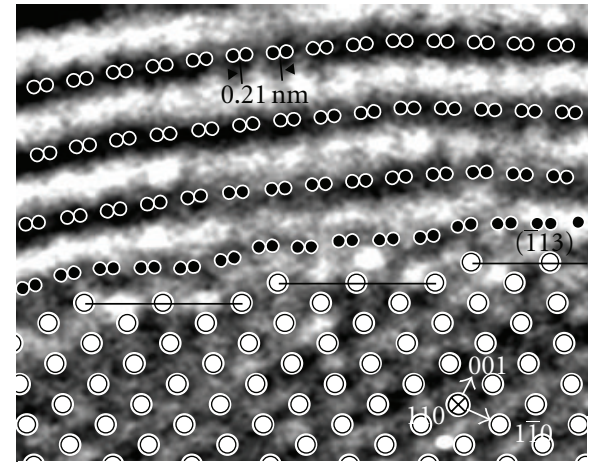

(b)

Figure 3: (a) High-resolution image of $(0001)_{\text {graphene }} /(\overline{1} 13)_{\text {Co }}$ interface in D-E region in the Co-encapsulated carbon nanocapsule shown in Figure 1(a). (b) The atomic configuration overlapping on the image in (a). The graphene layers are observed along $[\overline{1} \overline{1} 20]_{\text {graphene }}$.

TABLE 1: Orientation relationship between graphene and Co.

\begin{tabular}{|c|c|c|c|c|}
\hline Region & Orientational relationship & $\mathrm{d}(\mathrm{nm})$ & $\begin{array}{c}\text { axis }_{\mathrm{Co}} / /[\overline{1} \overline{1} 20]_{\text {graphene }} \\
\text { misfit }(\%)\end{array}$ & $\begin{array}{c}\text { axis }_{\mathrm{Co}} / /[\overline{1} 100]_{\text {graphene }} \\
\text { misfit }(\%)\end{array}$ \\
\hline$A-B$ & $(0001)[\overline{1} \overline{1} 20]_{\text {graphene }} / /(\overline{1} 1 \overline{1})[110]_{\mathrm{Co}}$ & $0.21 \pm 0.03$ & {$[110]_{\mathrm{Co}} 1.6$} & {$[\overline{1} 12]_{\mathrm{Co}} 1.8$} \\
\hline $\mathrm{B}-\mathrm{C}$ & $(0001)_{\text {graphene }} / /(\overline{1} 10)_{\mathrm{Co}}^{*}$ & $0.28 \pm 0.03$ & - & - \\
\hline C-D & $(0001)[\overline{1} \overline{1} 20]_{\text {graphene }} / /(\overline{1} 11)[110]_{\mathrm{Co}}$ & $0.30 \pm 0.05$ & {$[110]_{\mathrm{Co}} 1.6$} & {$[1 \overline{1} 2]_{\mathrm{Co}} 1.8$} \\
\hline D-E & $(0001)[\overline{1} \overline{1} 20]_{\text {graphene }} / /(\overline{1} 13)[110]_{\mathrm{Co}}$ & $0.21 \pm 0.03$ & $/ /$ & {$[3 \overline{3} 2]_{\mathrm{Co}} 49$} \\
\hline E-F & $(0001)[\overline{1} \overline{1} 20]_{\text {graphene }} / /(002)[110]_{\mathrm{Co}}$ & $0.21 \pm 0.03$ & // & {$[1 \overline{1} 0]_{\mathrm{Co}} 15$} \\
\hline
\end{tabular}

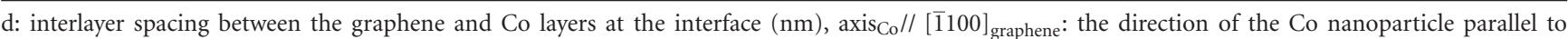
$[\overline{1} 100]_{\text {graphene }}$, misfit: the misfit between the graphene and Co layers along $[\overline{1} 100]_{\text {graphene. }}{ }^{*}$ Only layer/layer relationship was analyzed.

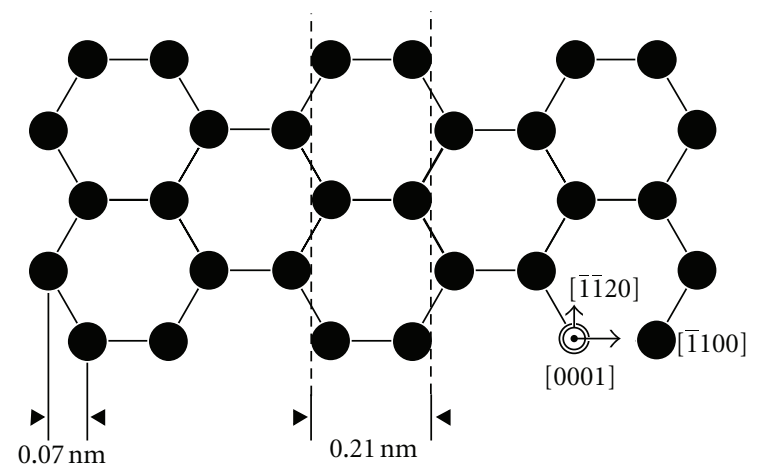

FIGURE 4: Atomic configuration of graphene layers, viewed along $[000 \overline{1}]_{\text {graphene }}$. The broken lines parallel to $[\overline{1} \overline{1} 20]_{\text {graphene }}$ indicate the positions of the black dots on the graphene layers in Figure 3(b).

viewed along $[\overline{1} \overline{1} 20]_{\text {graphene }}$ and $[110]_{\text {Co }}$ with details of the direction and the distances between the projected atomic columns is shown in Figure 5(d) to clear the orientational relationship and relative positions between the graphene layers and the Co nanoparticle. With similar reason, the atomic configuration along the direction perpendicular to the interfaces, that is, $[0001]_{\text {graphene }}$ and $[\overline{1} 13]_{\text {Co }}$ was shown in Figure 5(e). From the models in Figures 5(e) and 5(f), the relationship between the atomic configurations of the graphene layers and Co nanoparticle projected along the direction perpendicular to the interface can be analyzed. We found from these figures that a half of Co atoms locate around the on-top sites of carbon atoms on the interface in this region.

Figure 6 shows the orientational relationship between the graphene layers and Co nanoparticles at the interfaces in region A-B. The enlarged high-resolution image of the interface shown in Figure 1 is shown in Figure 6(a). We constructed the configuration model of carbon and Co atoms along the direction parallel to the interfaces. As in Figure 5(b), to confirm the matching of the lattice image and the model, the overlapping image of them is presented in Figure 6(b). The extracted atomic configuration is shown in Figure 6(c). The atomic configuration model at the interface viewed along $[\overline{1} \overline{1} 20]_{\text {graphene }}$ and $[110]_{\text {Co }}$ with details of the direction and the distances between the projected atomic columns is shown in Figure 6(d) to clear the orientational relationship and relative positions between the graphene layers and the Co crystal. With similar reason, the atomic configuration along the direction perpendicular to the interfaces, that is, $[0001]_{\text {graphene }}$ and $[\overline{1} 1 \overline{1}]_{\text {Co }}$ was shown in Figure 6(e). From the models in Figures 6(e) and 6(f), the overlapping of the atomic configurations of the graphene 


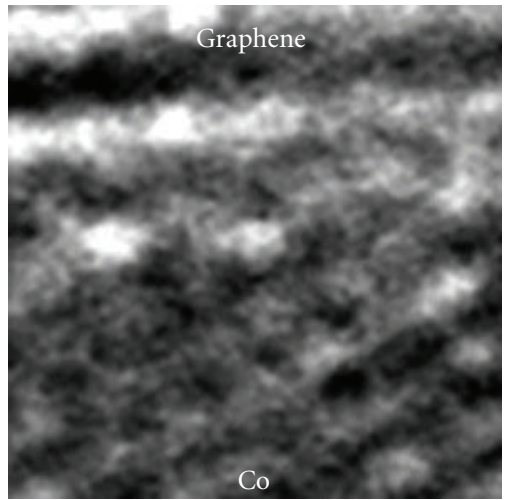

(a)

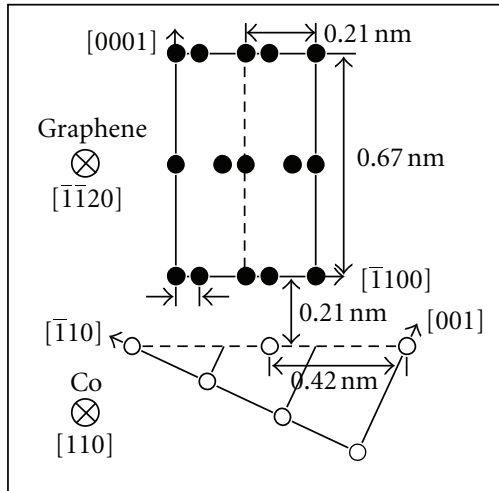

(d)

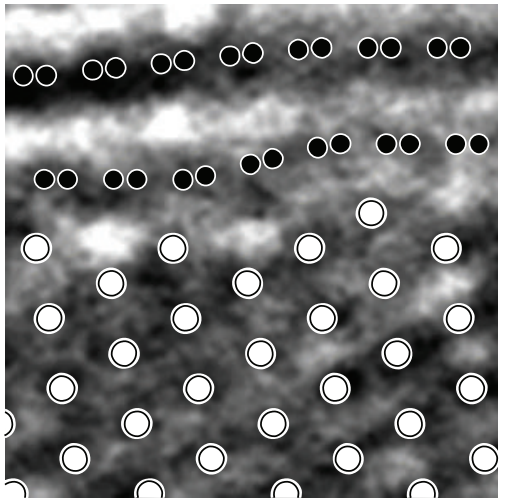

(b)
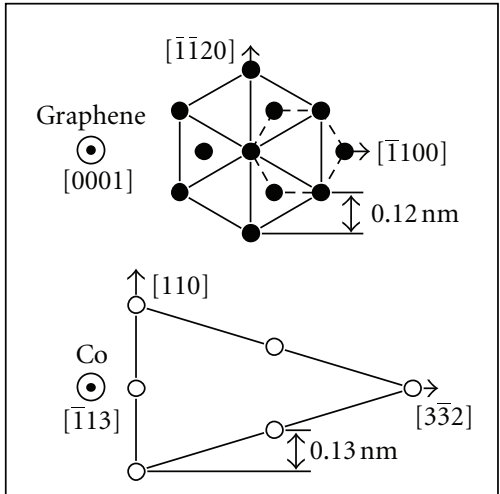

(e)

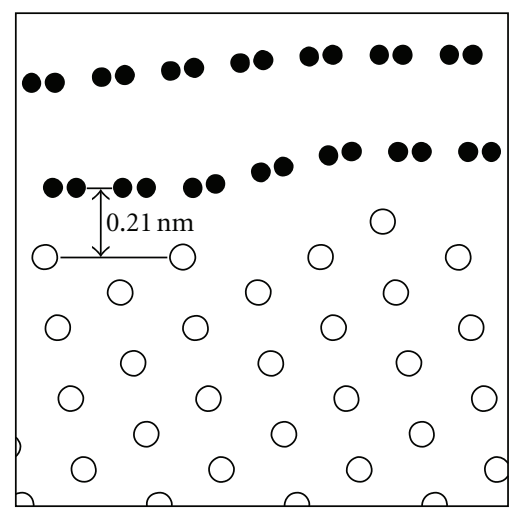

(c)

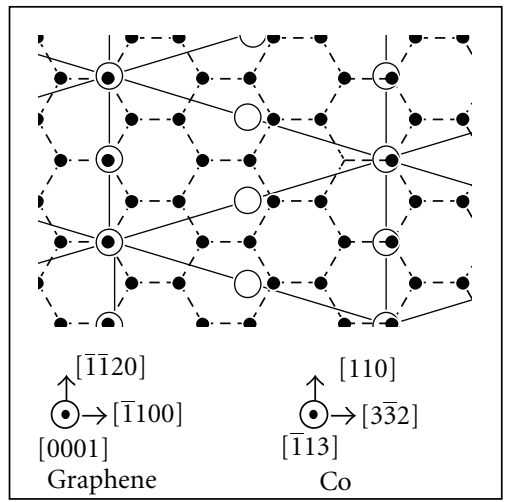

(f)

FIGURE 5: The orientational relationship between graphene layers and Co nanoparticle at the interface in region D-E shown in Figure 3. (a) Enlarged high-resolution image of the interface. (b) The atomic configuration overlapping on the image in the image in (a). (c) The extracted atomic configuration. Atomic configurations at the interface viewed along $[\overline{1} \overline{1} 20]_{\text {graphene }}$ and $[110]_{\mathrm{Co}}(\mathrm{d})$ and $[0001]_{\text {graphene }}$ and $[\overline{1} 13]_{\mathrm{Co}}(\mathrm{e})$. (f) Overlapping of the atomic configurations of the graphene layers and Co nanoparticle projected along the direction perpendicular to the interface, that is, $[0001]_{\text {graphene }}$ and $[\overline{1} 13]_{\mathrm{Co}}$. The filled and open circles indicate carbon and cobalt atoms.

layers and Co nanoparticle projected along the direction perpendicular to the interface can be considered. We found that most of Co atoms locate around the on-top sites of carbon atoms on the interface in this region.

The misfits at the interfaces in the five regions are shown in Table 1. In regions A-B, D-E, and E-F, the misfits at the interfaces along $[\overline{1} \overline{1} 20]_{\text {graphene }}$ are $1.6 \%$. On the other hand, the misfits along $[\overline{1} 100]_{\text {graphene }}$ are $1.8 \%, 49 \%$, and $15 \%$ for the three regions, respectively. The spacing between the graphene layer and the Co layer at the interfaces in the three regions ranges between the same range, that is, $0.21 \pm$ $0.03 \mathrm{~nm}$.

Figure 7 shows a $(0001)_{\text {graphene }} /(\overline{1} 10)_{\mathrm{Co}}$ interface in B-C region in the Co-encapsulated CNC shown in Figure 1(a). In this region, the configuration of $\mathrm{Co}$ atoms could be analyzed as in the regions $\mathrm{A}-\mathrm{B}$ and D-E. On the other hand, the configuration of carbon atoms cannot be identified because only line images are observed in the graphene region. Then, we found the interlayer spacing in this region, that is, $0.28 \mathrm{~nm}$.
Figure 8 shows a $(0001)_{\text {graphene }} /(\overline{1} 11)_{\mathrm{Co}}$ interface in C-D region in the Co-encapsulated CNC shown in Figure 1(a). As shown in Table 1, the orientational relationship in this region is equivalent to that in region A-B. However, the interlayer spacing in this region is $0.30 \pm 0.05 \mathrm{~nm}$, which is higher than that in region A-B. In Figure 8, the graphene layers at the interface bend around an additional atomic row on the Co facet. Strain is introduced in the graphene layers in $\mathrm{C}-\mathrm{D}$ region owing to the bending. This contributes to higher interlayer spacing.

\section{Discussion}

4.1. The Shape of the Graphene Layers at the Interfaces. The graphene layers at the interfaces were flat when the Co facets were flat, as shown in Figures 6 and 7. On the other hand, the graphene layers bent along the steps and additional atomic rows on the Co facets, as shown in Figures 5 and 8. Thus, the shape of graphene layers at the interfaces fit with the surface shape of the Co nanoparticle; a single graphene 


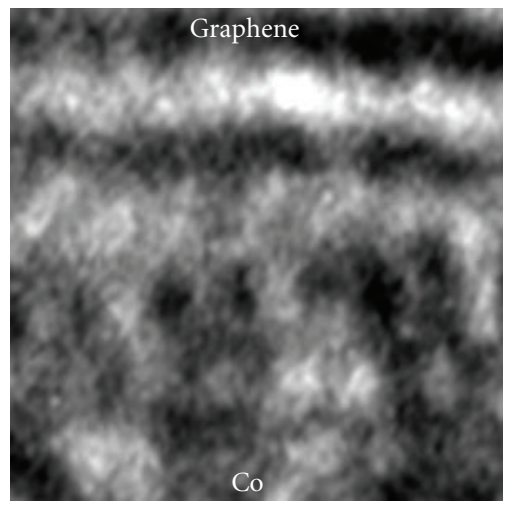

(a)

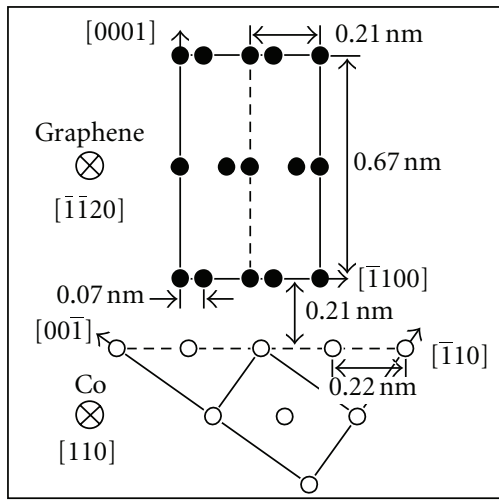

(d)

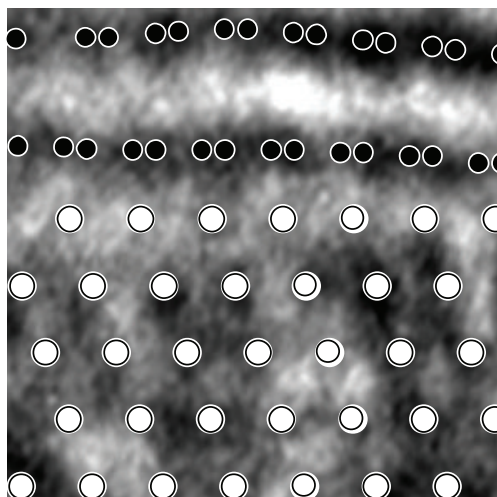

(b)

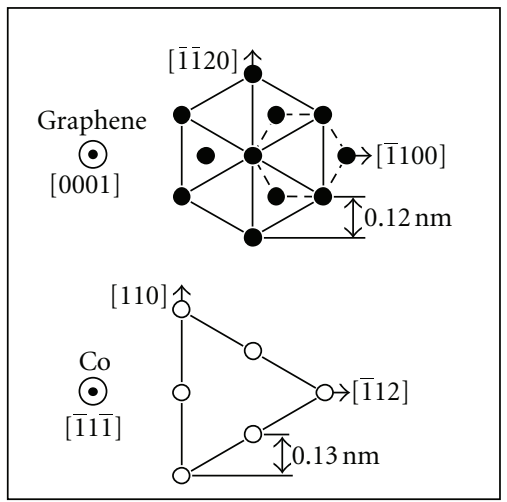

(e)

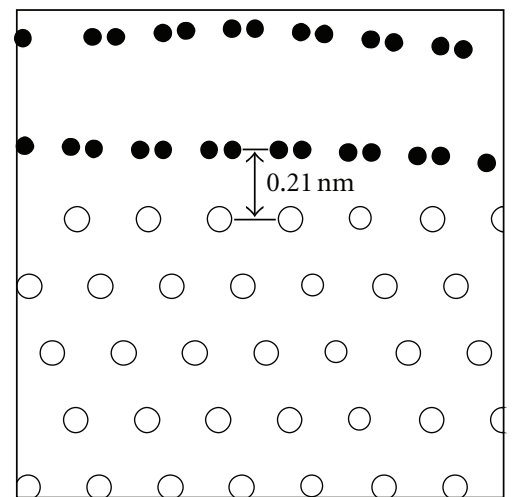

(c)

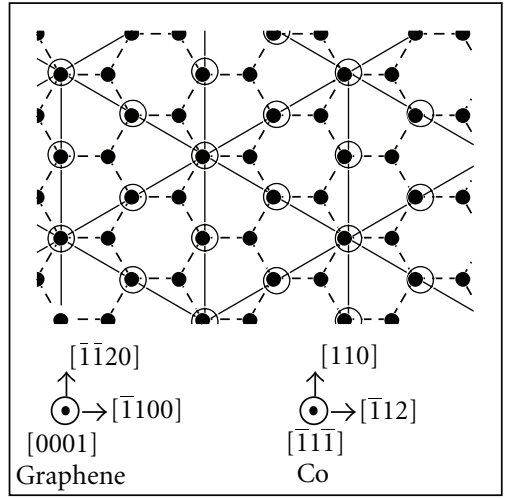

(f)

Figure 6: The orientational relationship at the $(0001)_{\text {graphene }} /(\overline{1} 1 \overline{1})_{\mathrm{Co}}$ interface in region A-B shown in Figure 1. (a) Enlarged high-resolution image. (b) The atomic configuration overlapping on the image in (a). (c) The extracted atomic configuration. Atomic configurations at the interface viewed along $[\overline{1} \overline{1} 20]_{\text {graphene }}$ and $[110]_{\mathrm{Co}}(\mathrm{d})$ and $[0001]_{\text {graphene }}$ and $[\overline{1} 1 \overline{1}]_{\mathrm{Co}}$ (e). (f) Overlapping of the atomic configurations of the graphene layers and Co nanoparticle projected along the direction perpendicular to the interface, i.e., $[0001]_{\text {graphene }}$ and $[\overline{1} 1 \overline{1}]_{\mathrm{Co}}$. The filled and open circles indicate carbon and cobalt atoms.

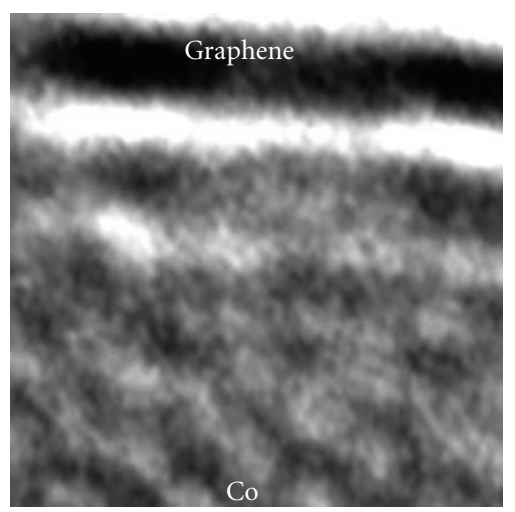

(a)

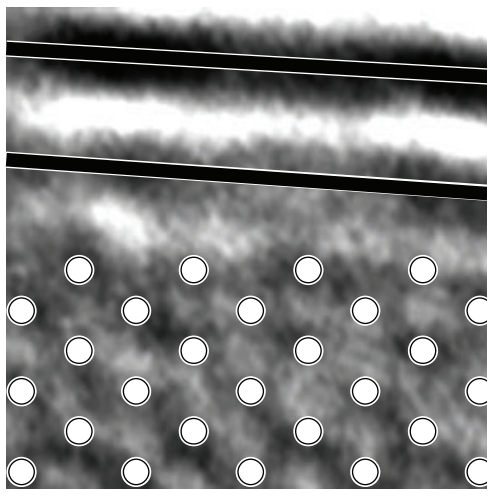

(b)

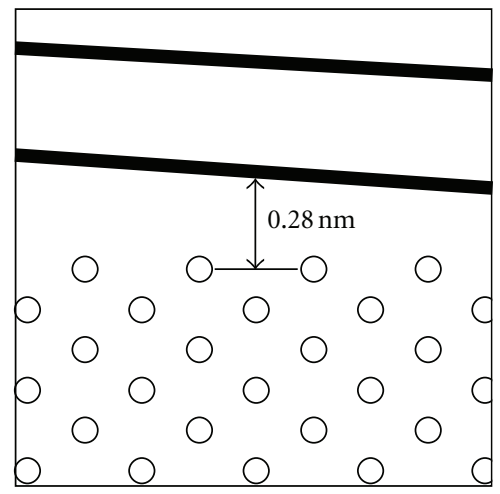

(c)

Figure 7: (a) Enlarged high-resolution image of the $(0001)_{\text {graphene }} /(\overline{1} 10)_{\text {Co }}$ interface in region B-C shown in Figure 1 . (b) The atomic configuration overlapping on the image in (a). (c) The extracted atomic configuration. The atomic position on the graphene layers and the direction of the graphene layers are not identified. 


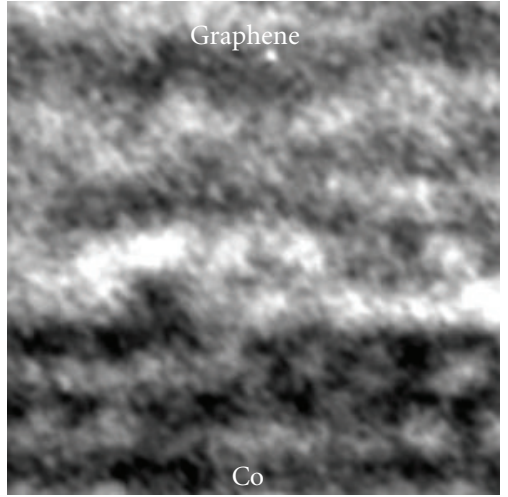

(a)

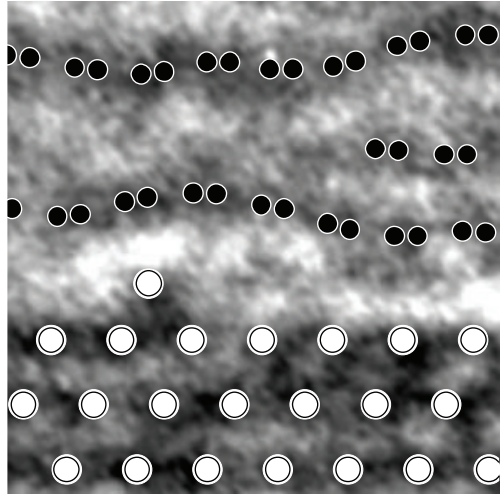

(b)

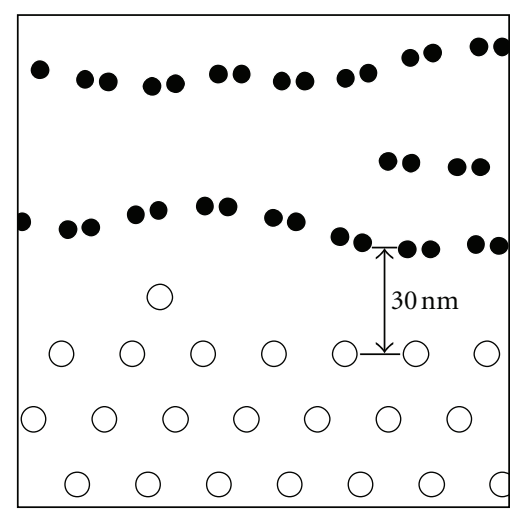

(c)

FIgURE 8: (a) Enlarged high-resolution image of the $(0001)_{\text {graphene }} /(\overline{1} 11)_{\text {Co }}$ interface in C-D region shown in Figure 1. (b) The atomic configuration overlapping on the image in (a). (c) The extracted atomic configuration.

layer covers the Co facet even if the Co facets are stepped and curved. In the gas-evaporation, first, $\mathrm{Co} / \mathrm{carbon}$ solid solution phases are formed by mixture of atomized Co and carbon atoms at high temperature. The solid solubility of carbon in Co decreases during cooling. From this, it is proposed that the solved carbon precipitates as graphene layers on the Co nanoparticles at lower temperatures [15]. In such precipitation, the graphene layers are conformed to the Co facets.

4.2. The Graphene/Co Interlayer Spacing at the Interfaces. Although the misfit along $[\overline{1} 100]_{\text {graphene }}$ is different in regions $\mathrm{A}-\mathrm{B}, \mathrm{D}-\mathrm{E}$, and E-F, the spacing between the graphene layer and the Co layer at the interfaces ranges between the same range. Interface steps were observed in region $\mathrm{D}$-E, whereas flat interfaces were observed in regions A-B and E-F. For the $(0001)_{\text {graphene }} /(\overline{1} 11)_{\mathrm{Co}}$ interface showing the largest interlayer spacing, the misfits along $[\overline{1} 100]_{\text {graphene }}$ and $[\overline{1} \overline{1} 20]_{\text {graphene }}$ are similar to those in A-B region. Thus, the range of the observed interlayer spacing is not necessarily influenced by misfits and interface steps. Swart et al. estimated the interlayer spacing at a $(0001)_{\text {graphene }} /(111)_{\text {Co }}$ interface to be $0.215 \mathrm{~nm}$ by periodic slab calculations [29]. This calculated value is smaller than that of the interface with the same orientational relationship (region C-D), whereas it is similar to that in different relationships (regions D-E and E-F). As shown in Figure 1, considerable strains were introduced in the graphene layers around point $\mathrm{C}$, that is, around regions $\mathrm{B}-\mathrm{C}$ and $\mathrm{C}-\mathrm{D}$. In these regions, the interlayer spacing was $0.28-0.30 \mathrm{~nm}$. Thus, it is suggested that the larger interlayer spacing are caused by strain in the graphene layers.

\section{Conclusion}

The graphene/Co interfaces in Co-encapsulated CNCs were observed by high-resolution TEM. The atomic configuration and the interlayer spacing at the graphene/Co interfaces were investigated. The observed interlayer spacing between $0.21 \pm$ $0.03 \mathrm{~nm}$ at the graphene/Co interface was observed regardless of the misfit and interface steps. On the other hand, the larger spacing between 0.28 to $0.30 \mathrm{~nm}$ was observed around considerably strained graphene layers.

\section{Acknowledgments}

This study was partly supported by Grants-in-Aid from the Ministry of Education, Culture, Sport, Science, and Technology, Japan (nos. 22310065 and 23651127).

\section{References}

[1] H. W. Kroto, J. R. Heath, S. C. O’Brien, R. F. Curl, and R. E. Smalley, "C $\mathrm{C}_{60}$ : buckminsterfullerene," Nature, vol. 318, no. 6042, pp. 162-163, 1985.

[2] S. Iijima, "Helical microtubules of graphitic carbon," Nature, vol. 354, no. 6348, pp. 56-58, 1991.

[3] T. W. Ebbesen and P. M. Ajayan, "Large-scale synthesis of carbon nanotubes," Nature, vol. 358, no. 6383, pp. 220-222, 1992.

[4] D. Ugarte, "Curling and closure of graphitic networks under electron-beam irradiation," Nature, vol. 359, no. 6397, pp. 707-709, 1992.

[5] D. S. Bethune, C. H. Kiang, M. S. De Vries et al., "Cobaltcatalysed growth of carbon nanotubes with single-atomiclayer walls," Nature, vol. 363, no. 6430, pp. 605-607, 1993.

[6] R. D. Heidenreich, W. M. Hess, and L. L. Ban, "A test object and criteria for high resolution electron microscopy," Journal of Applied Crystallography, vol. 1, no. 1, pp. 1-19, 1968.

[7] Y. Saito, T. Yoshikawa, M. Inagaki, M. Tomita, and T. Hayashi, "Growth and structure of graphitic tubules and polyhedral particles in arc-discharge," Chemical Physics Letters, vol. 204, no. 3-4, pp. 277-282, 1993.

[8] Y. Saito, T. Yoshikawa, M. Okuda et al., "Carbon nanocapsules encaging metals and carbides," Journal of Physics and Chemistry of Solids, vol. 54, no. 12, pp. 1849-1860, 1993.

[9] F. Banhart, P. Redlich, and P. M. Ajayan, "Irradiation effects in carbon nanostructures," Chemical Physics Letters, vol. 292, no. 4-6, pp. 554-560, 1998.

[10] V. Z. Mordkovich, A. G. Umnov, T. Inoshita, and M. Endo, "The observation of multiwall fullerenes in thermally treated 
laser pyrolysis carbon blacks," Carbon, vol. 37, no. 11, pp. 1855-1858, 1999.

[11] S. Iijima, "Direct observation of the tetrahedral bonding in graphitized carbon black by high resolution electron microscopy," Journal of Crystal Growth, vol. 50, no. 3, pp. 675$683,1980$.

[12] R. S. Ruoff, D. C. Lorents, B. Chan, R. Malhotra, and S. Subramoney, "Single crystal metals encapsulated in carbon nanoparticles," Science, vol. 259, no. 5093, pp. 346-348, 1993.

[13] M. Tomita, Y. Saito, and T. Hayashi, " $\mathrm{LaC}_{2}$ encapsulated in graphite nano-particle," Japanese Journal of Applied Physics, vol. 32, no. 2B, pp. L280-L282, 1993.

[14] T. Hihara, H. Onodera, K. Sumiyama et al., "Magnetic properties of iron in nanocapsules," Japanese Journal of Applied Physics, vol. 33, no. 1A, pp. L24-L25, 1994.

[15] Y. Saito, "Nanoparticles and filled nanocapsules," Carbon, vol. 33, no. 7, pp. 979-988, 1995.

[16] T. Oku and K. Suganuma, "Carbon nanocage structures formed by one-dimensional self-organization of gold nanoparticles," Chemical Communications, no. 23, pp. 2355-2356, 1999.

[17] A. Vinu, M. Miyahara, V. Sivamurugan, T. Mori, and K. Ariga, "Large pore cage type mesoporous carbon, carbon nanocage: a superior adsorbent for biomaterials," Journal of Materials Chemistry, vol. 15, no. 48, pp. 5122-5127, 2005.

[18] J.-S. Yu, S. B. Yoon, Y. J. Lee, and K. B. Yoon, "Fabrication of bimodal porous silicate with silicalite-1 core/mesoporous shell structures and synthesis of nonspherical carbon and silica nanocases with hollow core/mesoporous shell structures," The Journal of Physical Chemistry B, vol. 109, no. 15, pp. 70407045, 2005.

[19] J. N. Wang, L. Zhang, J. J. Niu et al., "Synthesis of high surface area, water-dispersible graphitic carbon nanocages by an in situ template approach," Chemistry of Materials, vol. 19, no. 3, pp. 453-459, 2007.

[20] R. Rosei, M. De Crescenzi, F. Sette, C. Quaresima, A. Savoia, and P. Perfetti, "Structure of graphitic carbon on $\mathrm{Ni}(111)$ : a surface extended-energy-loss fine-structure study," Physical Review B, vol. 28, no. 2, pp. 1161-1164, 1983.

[21] R. Rosei, S. Modesti, F. Sette, C. Quaresima, A. Savoia, and P. Perfetti, "Electronic structure of carbidic and graphitic carbon on Ni(111)," Physical Review B, vol. 29, no. 6, pp. 3416-3422, 1984.

[22] A. Nagashima, N. Tejima, and C. Oshima, "Electronic states of the pristine and alkali-metal-intercalated monolayer graphite/Ni(111) systems," Physical Review B, vol. 50, no. 23, pp. 17487-17495, 1994.

[23] Y. Gamo, A. Nagashima, M. Wakabayashi, M. Terai, and C. Oshima, "Atomic structure of monolayer graphite formed on Ni(111)," Surface Science, vol. 374, no. 1-3, pp. 61-64, 1997.

[24] F. Abild-Pedersen, J. K. Nørskov, J. R. Rostrup-Nielsen, J. Sehested, and S. Helveg, "Mechanisms for catalytic carbon nanofiber growth studied by $a b$ initio density functional theory calculations," Physical Review B, vol. 73, no. 11, Article ID 115419, pp. 1-13, 2006.

[25] G. Kalibaeva, R. Vuilleumier, S. Meloni, A. Alavi, G. Ciccotti, and R. Rosei, " $A b$ initio simulation of carbon clustering on an $\mathrm{Ni}(111)$ surface: a model of the poisoning of nickel-based catalysts," The Journal of Physical Chemistry B, vol. 110, no. 8, pp. 3638-3646, 2006.

[26] S.-G. Wang, X. Y. Liao, D. B. Cao, Y. W. Li, J. Wang, and H. Jiao, "Formation of carbon species on $\mathrm{Ni}(111)$ : structure and stability," The Journal of Physical Chemistry C, vol. 111, no. 29, pp. 10894-10903, 2007.

[27] G. Giovannetti, P. A. Khomyakov, G. Brocks, V. M. Karpan, J. Van Den Brink, and P. J. Kelly, "Doping graphene with metal contacts," Physical Review Letters, vol. 101, no. 2, Article ID 026803, 2008.

[28] A. Grüneis and D. V. Vyalikh, "Tunable hybridization between electronic states of graphene and a metal surface," Physical Review B, vol. 77, no. 19, Article ID 193401, 2008.

[29] J. C. W. Swart, E. Van Steen, I. M. Ciobícă, and R. A. Van Santen, "Interaction of graphene with FCC-Co(111)," Physical Chemistry Chemical Physics, vol. 11, no. 5, pp. 803-807, 2009.

[30] Y. Saito, T. Yoshikawa, M. Okuda et al., "Carbon nanocapsules encaging metals and carbides," Journal of Physics and Chemistry of Solids, vol. 54, no. 12, pp. 1849-1860, 1993.

[31] J. Jiao, S. Seraphin, X. Wang, and J. C. Withers, "Preparation and properties of ferromagnetic carbon-coated $\mathrm{Fe}, \mathrm{Co}$, and $\mathrm{Ni}$ nanoparticles," Journal of Applied Physics, vol. 80, no. 1, pp. 103-108, 1996. 

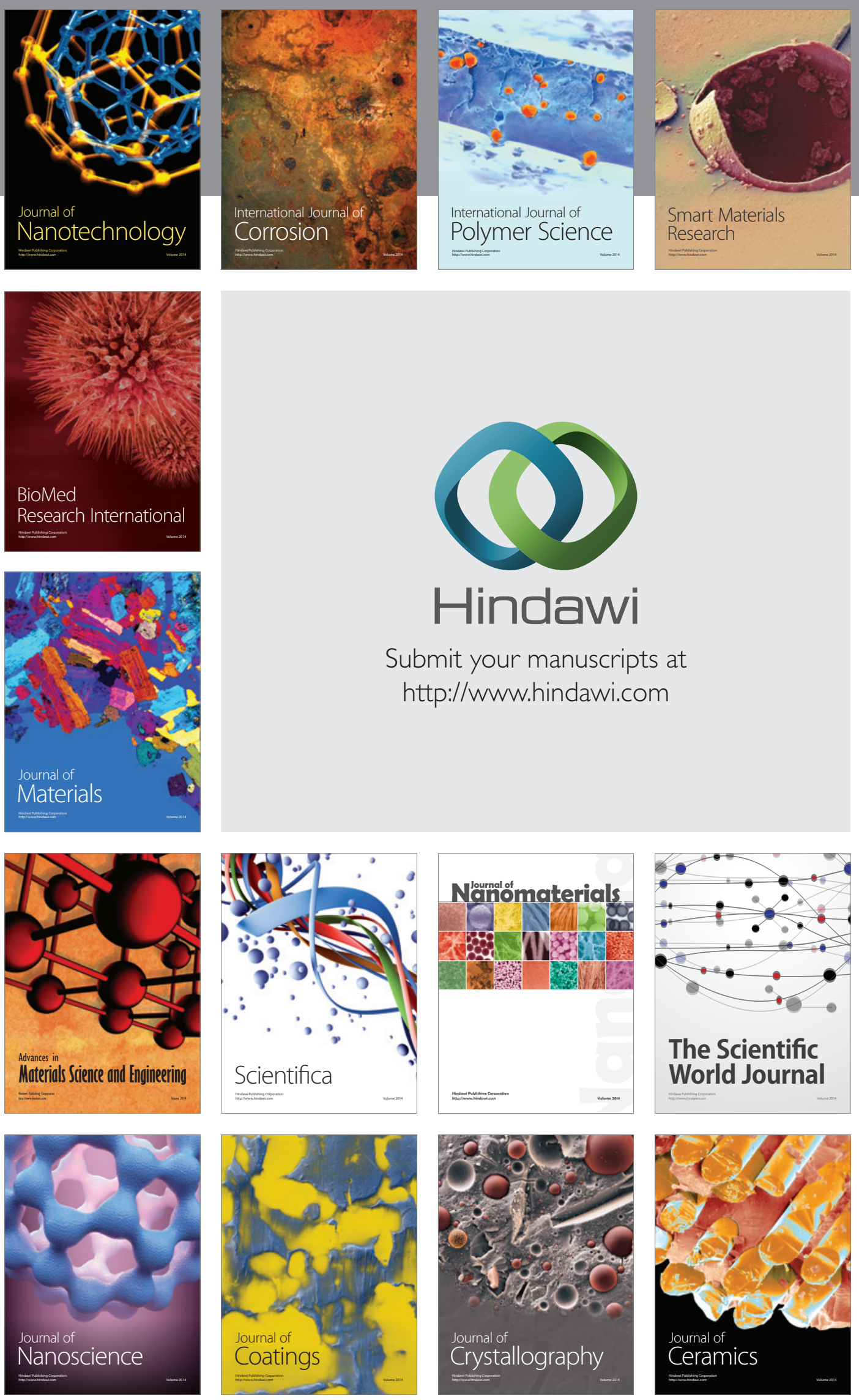

The Scientific World Journal

Submit your manuscripts at

http://www.hindawi.com

\section{World Journal}

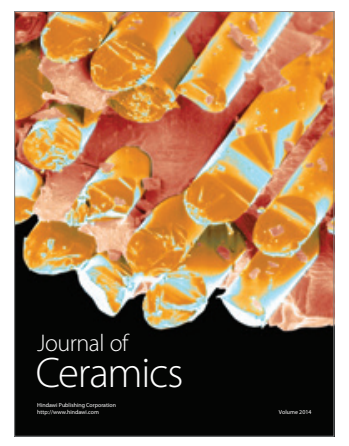

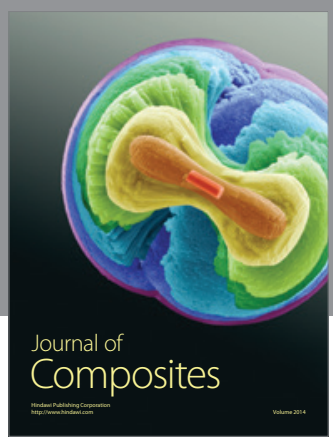
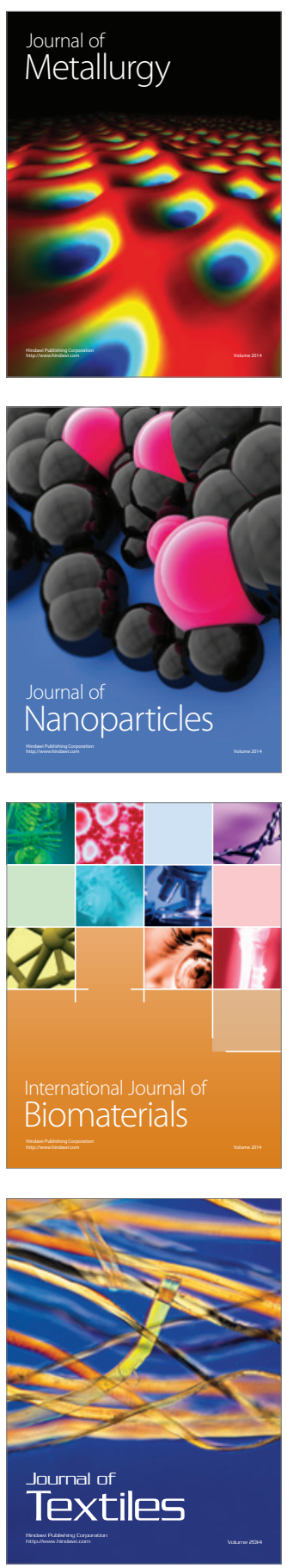